be equal by the Cauchy integral theorem (by [1] and a result in the author's dissertation not yet published).

Theorem 1 follows directly from Theorem 3.

\title{
BIBLIOGRAPHY
}

1. E. R. Lorch, The theory of analytic functions in normed abelian vector rings, Trans. Amer. Math. Soc. vol. 54 (1943) pp. 414-425.

2. L. Pontrjagin, Topological groups, Princeton University Press, 1946, p. 231.

3. M. Nagumo, Einige analytische Untersuchungen in linearen metrischen Ringen, Jap J. Math. (1936).

Columbia University and

UNIVERSITY OF MARYLAND

\section{REMARK ON A FORMULA FOR THE BERNOULLI NUMBERS}

\section{CARLITZ}

Some years ago Garabedian [1] proved the following formula:

$$
B_{k+1}=\frac{(-1)^{k+1}(k+1)}{2^{k+1}-1} \sum_{r=0}^{k}(-1)^{r} \frac{\Delta^{r} 1^{k}}{2^{r+1}} \quad(k \geqq 0),
$$

where the even suffix notation is employed for the Bernoulli numbers. The proof of (1) made use of the sum of a certain divergent series.

We wish to point out that (1) is not new. It can be found (in somewhat different notation) in $[3$, p. 224 , formula (68)].

It may be of interest to give a short proof of (1). We use the formula $[2$, p. 28]

$$
C_{k}=2^{k+1}\left(1-2^{k+1}\right) \frac{B_{k+1}}{k+1},
$$

where the $C_{k}$ are the coefficients in the Euler polynomial:

$$
E_{k}(x)=\left(x+\frac{C}{2}\right)^{k}=\sum_{s=0}^{k}\left(\begin{array}{l}
k \\
s
\end{array}\right) 2^{-s} C_{s} x^{k-s} .
$$

Then in view of

$$
E_{k}(x+1)+E_{k}(x)=2 x^{k},
$$

we have

$$
E_{k}(x)=\left(1+\frac{1}{2} \Delta\right)^{-1} x^{k}=\sum_{s=0}^{k}(-1)^{\cdot} 2^{-s} \Delta^{s} x^{k}
$$

Received by the editors September 5, 1952. 
If we take $x=1$ in (5) and use (3) and (4), we get

$$
C_{k}=-2^{k} E_{k}(1)=-\sum_{s=0}^{k}(-1)^{\cdot} \cdot 2^{k-s} \Delta^{\cdot} 1^{k} \text {. }
$$

Substitution in (2) leads at once to (1).

In a similar way we can prove

$$
\begin{gathered}
B_{k+1}=\frac{(-1)^{k+1}(k+1)}{2^{k+1}-1} \sum_{r=0}^{k}(-1)^{r} 2^{-r-1} \Delta^{r} 0^{k} . \\
\text { REFERENCES }
\end{gathered}
$$

1. H. L. Garabedian, $A$ new formula for the Bernoulli numbers, Bull. Amer. Math. Soc. vol. 46 (1940) pp. 531-533.

2. N. E. Nörlund, Vorlesungen über Differenzenrechnung, Berlin, 1924.

3. J. Worpitzky, Studien über die Bernoullischen und Eulerschen Zahlen, J. Reine Angew. Math. vol. 94 (1883) pp. 203-232.

DUKe UNIVERSITY 